\title{
Membrane physiology and biophysics in the next decade: an open balcony to multiple scenarios
}

\author{
Mario L. Díaz* \\ Universidad de La Laguna, Tenerife, Spain \\ *Correspondence: madiaz@ull.es
}

No doubt the cell membrane represents the first evolutionary milestone in the construction of life. The basic structure of primitive membranes likely provided a rather simple barrier to isolate an intracellular compartment, where molecular "protobricks" started (assisted or not) to develop life, a process that lasted around a 1000 million years. However, membrane entities dramatically changed upon evolution of metazoans, paralleling the appearance of differentiated tissues and organs. In modern organisms, the differences in cell membrane composition reflect neatly these evolutionary changes: supporting specialized functions, and acquiring more and more molecular complexity as cells within tissues and organs became more and more specialized. Countless symphonies in animal cell life rely on plasma membrane composition and integrity, and examples of membrane adaptation to enable physiological adaptation to different physical environments are spectacular (Hazel and Williams, 1990).

The development of high-resolution electrophysiological techniques in the 1980's has opened a revolutionary window for the study and comprehension of ion channels, allowing the unprecedented real-time observation of single protein molecules in action. The discovery of the patch-clamp technique represented a real milestone and gave rise to a frenzied scientific renaissance. Nearly at the same time, the sophistication of molecular biology techniques allowed the cloning of the first ion channels, the voltage-dependent sodium channel and the nicotinic acetylcholine receptor, both from Torpedo californica (Numa, 1986). Since then our current understanding on how ion channels work and their contribution to physiological functions has increased enormously. However, the answers to those former questions have given birth to new enigmas, i.e., ion channels are not that different from ion transporters, and the clear functional separation between both entities is becoming fainter. As an example, the $\mathrm{ClC}$ family of ion channels shows an unusually broad variety of functional behaviors, as some members work as gated chloride channels and others as secondary chloride transporters, for instance ClC-4 and ClC-5 anion channels may be $\mathrm{Cl}^{-} / \mathrm{H}^{+}$-exchangers (Jentsch, 2008). Some other ion channels, like the voltage-dependent anion channels characteristic of mitochondrial outer membrane but also present at the plasma membrane, can exhibit different conductance states with very different permeabilities. This channel is permeable to anions, including bulky anionic metabolites (such as ATP) in the largest conductance open state, but behaves as a cation-selective channel in the closed state. In addition, it can also function as a membrane-bound redox enzyme under specific conditions (Shoshan-Barmatz et al., 2010). The present panorama with ion channels is certainly complex and requires extensive (re)investigation.

One of the most stimulating issues in the study of cell membrane is the number of human and animal pathologies that are linked to alterations in discrete membrane components, these including ion channels, hormone and neurotransmitter receptors, transporters, membrane-associated enzymes and membrane lipids. The spectrum of these diseases encompasses a wide range of examples that extend from cystic fibrosis to long QT syndrome, from nephrolithiasis to hypertension, and from malignant hyperthermia to neurodegeneration. Defects in membrane components have received considerable attention in the last several years and, to some extent, part of these defects can be explained by mutated or altered membrane proteins. Yet, in many other cases, the biochemical defects can not be proven to be genetically derived, but rather are associated with abnormal protein processing, folding, association or altered protein trafficking toward the membrane (Kass, 2005). Therefore, it is vital that we further our understanding of the various abnormal forms and processing of proteins that are generated during the development of disease; this will certainly aid the design of appropriate therapeutic strategies.

The complexity of the membrane scenario is even more puzzling when it is realized that non-cognate molecules physically associate with it. This is the case of canonical steroid receptors, such estrogen or progesterone receptors that, though lacking alpha helix domains, appear to be tightly integrated and immunoprecipitate with prototypical membrane proteins, i.e., caveolin, in purified plasma membranes. The interesting point around this association is that their activation by cognate ligands triggers intracellular signals that largely differ from their classical transcriptional activities, which have given rise to a new concept on steroid transduction "Membrane-initiated steroid signaling" (Nemere et al., 2003). Nonetheless, despite considerable molecular evidence demonstrating the widespread nature of this phenomenon, the physiological relevance of this observation at normal circulating levels of steroids is extremely limited, and much work is still to be done.

Sophisticated imaging techniques, such as confocal microscopy, TIRF (Total Internal Reflection Fluorescence), FRET (Förster resonance energy transfer) or multiphoton laser scanning microscopy have been developed in the last decades to study membrane biology in living cells, and the results have undoubtedly revealed that cell membranes are highly dynamic structures experiencing a continuous dialog and crosstalk with intracellular membranes and the cytoskeleton. The most recent research shows that cell membranes are by far different from homogeneous as initially proposed by Singer and Nicolson (1972), but consisting of complex mosaics of different microdomains, each containing different clusters of proteins and lipids, that segregates laterally within the vast sea of plasma membrane. At least some of these domains, namely lipid rafts, are known to function as signaling 
platforms involved in cellular communication and transduction. What causes this segregation and what mechanisms are responsible for maintaining this topological organization under physiological conditions is a matter of intense investigation and represents a central challenge for the comprehension of membrane function.

Plasma membranes not only exhibit horizontal heterogeneity, but also display transbilayer asymmetry. This is obvious for extrinsic membrane proteins, but what makes this surprising is that it is also reflected in the distribution of membrane phospholipids and cholesterol, which makes the inner leaflet of the bilayer different than that as seen from the extracellular medium. At present, we have only taken more or less detailed pictures of live cell membranes but the physiological meanings of these structural complexities remain mysterious. The observation that membrane asymmetry is disrupted in some human diseases, i.e., Alzheimer's disease, indicates that maintaining membrane structure is crucial for normal neuronal function. We still need to explore the mechanisms that lead to these alterations, and their precise involvement in the origin and progression of the pathologies.

One of the most important limitations in the comprehension of membrane protein structure-function relationship emerges from the fact that, unlike cytosolic proteins, membrane-embedded proteins are not readily amenable to existing crystallization methods and only few protein structures have now been determined using $\mathrm{X}$-ray crystallography. This is due to the fact that membrane proteins require a balanced hydrophilic and lipophilic environment, while most protein chemistry methods work mainly in water-based media. There is an urgent need for advances in technologies to generate structural and functional information for membrane proteins. Despite notable technological developments, obstacles related to extraction and solubilization of membrane proteins is encountered, and alternative methods are needed to allow proteomic applications, mainly gel-based analyses and mass spectrometry (Rabilloud, 2009). Proteomic analysis of membrane proteins is a promising approach for the identification of novel drug targets and disease biomarkers. In the search for novel membrane proteins, analysis of protein sequences using computational and bioinformatic tools will allow for the prediction of the presence of transmembrane integratable domains. Along with technological innovations in the foreseeable future, advances in the areas of sample preparation and computational prediction of membrane proteins will lead to exciting discoveries.

Another important issue in the field of membrane physiology research is that we need to understand protein-lipid interactions within membrane domains. It is known that, at least for some integral proteins, there is an absolute requirement of a specific microenvironment. In addition, peripheral membrane proteins that contain hydrophobic anchors, i.e., glycosylphosphatidylinositol (GPI)-anchored proteins that penetrate the interfacial region of the membrane in a domain specific manner need a particular lipid composition. Experimental evidence indicates that alteration of the lipid environment critically affects the thermodynamic properties of membrane proteins, likely due to alteration of conformational changes during the catalytic cycle of the particular protein. We obtained this knowledge, from ion channels reconstituted in liposomes: a particular phospholipid composition determines a particular behavior. It is obvious then the necessity to combine information from biochemical, molecular physiology and biophysical approaches to achieve a real view on how proteins and lipids interact at the plasma membrane and modulate membrane function. Moreover, tackling these studies could help us to gain knowledge on the cellular basis of membrane-associated pathologies that lacks a known genetic background.
In conclusion, the impressive research and knowledge accumulated in the last decades in the field of membrane physiology and biophysics have uniquely positioned us to explore the multiple facets of cell membrane functions in health and disease. It is fascinating to realize how a few nanometers of life can hold such enormous entropy. It is the grand challenge of this specialty journal to integrate all this information in the puzzle of cell biology in physiology and medicine. Exciting times wait ahead.

\section{REFERENCES}

Hazel, J. R., and Williams, E. E. (1990). The role of alterations in membrane lipid composition in enabling physiological adaptation of organisms to their physical environment. Prog. Lipid Res. 29, 167-227.

Jentsch, T.J. (2008). CLC chloride channels and transporters: from genes to protein structure, pathology and physiology. Crit. Rev. Biochem. Mol. Biol. 43, 3-36.

Kass, R. S. (2005). The channelopathies: novel insights into molecular and genetic mechanisms of human disease. J. Clin. Invest. 115, 1986-1989.

Nemere, I., Pietras, R. J., and Blackmore, P. F. (2003). Membrane receptors for steroid hormones: signal transduction and physiological significance. J. Cell. Biochem. 88, 438-445.

Numa, S. (1986). Molecular basis for the function of ionic channels. Biochem. Soc. Symp. 52, 119-143.

Rabilloud, T. (2009). Membrane proteins and proteomics: love is possible, but so difficult. Electrophoresis 30(Suppl. 1), S174-S180.

Shoshan-Barmatz, V., De Pinto, V., Zweckstetter, M., Raviv, Z., Keinan, N., and Arbel, N. (2010). VDAC, a multi-functional mitochondrial protein regulating cell life and death. Mol. Aspects Med. 31, 227-285.

Singer, S. J., and Nicolson, G. L. (1972). The fluid mosaic model of the structure of cell membranes. Science $175,720-731$

Received: 13 July 2010; accepted: 13 July 2010; published online: 24 August 2010.

Citation: Diaz ML (2010) Membrane physiology and biophysics in the next decade: an open balcony to multiple scenarios. Front. Physio. 1:23. doi: 10.3389/ fphys.2010.00023

This article was submitted to Frontiers in Membrane Physiology and Biophysics, a specialty of Frontiers in Physiology.

Copyright (C) 2010 Díaz. This is an open-access article subject to an exclusive license agreement between the authors and the Frontiers Research Foundation, which permits unrestricted use, distribution, and reproduction in any medium, provided the original authors and source are credited. 